\title{
Characterization of a high-power tapered semiconductor amplifier system
}

\author{
D. Voigt, E.C. Schilder, R.J.C. Spreeuw, and H.B. van Linden van den Heuvell \\ Van der Waals-Zeeman Instituut, Universiteit van Amsterdam, \\ Valckenierstraat 65, 1018 XE Amsterdam, the Netherlands \\ e-mail:voigt@wins.uva.nl \\ (May 31, 2018)
}

We have characterized a semiconductor amplifier laser system which provides up to $200 \mathrm{~mW}$ output after a single-mode optical fiber at $780 \mathrm{~nm}$ wavelength. The system is based on a tapered semiconductor gain element, which amplifies the output of a narrow-linewidth diode laser. Gain and saturation are discussed as a function of operating temperature and injection current. The spectral properties of the amplifier are investigated with a grating spectrometer. Amplified spontaneous emission (ASE) causes a spectral background with a width of $4 \mathrm{~nm}$ FWHM. The ASE background was suppressed to below our detection limit by a proper choice of operating current and temperature, and by sending the light through a single-mode optical fiber. The final ASE spectral density was less than $0.1 \mathrm{nW} / \mathrm{MHz}$, i.e. less than $0.2 \%$ of the optical power. Related to an optical transition linewidth of $\Gamma / 2 \pi=6 \mathrm{MHz}$ for rubidium, this gives a background suppression of better than $-82 \mathrm{~dB}$. An indication of the beam quality is provided by the fiber coupling efficiency up to $59 \%$. The application of the amplifier system as a laser source for atom optical experiments is discussed.

42.55.Px: Semiconductor lasers; laser diodes

42.60.Da: Resonators, cavities, amplifiers, arrays, and rings

32.80.Pj: Optical cooling of atoms; trapping

\section{INTRODUCTION}

The techniques of laser cooling and trapping of neutral atoms [i] require stable, narrow linewidth and frequency tunable laser sources. Commonly used systems for the near infrared wavelengths are based on external grating diode lasers (EGDL) [2]. Optical feedback from a grating narrows the linewidth to less than $1 \mathrm{MHz}$ and provides tunability. High-power single transverse mode diode lasers can provide up to $80 \mathrm{~mW}$ optical output at wavelengths below $800 \mathrm{~nm}$. In this power range diode lasers thus provide a less costly alternative to Ti:Sapphire lasers.

If more power is required, the output of an EGDL can be amplified. Presently, there are three common techniques based on semiconductor gain elements: (i) Injection-locking of a single-mode laser diode [3] by seeding light from an EGDL results typically in $60-80 \mathrm{~mW}$ optical power at $780 \mathrm{~nm}$ wavelength. (ii) Amplification in a double-pass through a broad-area emitting diode laser (BAL) [- [7]. This yields an optical output of typically $150 \mathrm{~mW}$ after spatial filtering. A disadvantage is the relatively low gain of 10-15, requiring high seed input power.
The BAL gain can be improved using phase conjugating mirrors in the seed incoupling setup [8]. (iii) Travellingwave amplification in a semiconductor gain element with a tapered waveguide (TA) [5, 9, 10]. Compared to a BAL this yields higher gain and higher power after spatial filtering. This approach requires much lower input and a less complex optical setup than a BAL. However, a TA gain element is considerably more expensive.

In this work we investigate a TA system that amplifies the narrow-linewidth seed beam of an EGDL and provides up to $200 \mathrm{~mW}$ optical output from a singlemode optical fiber. We operate the system on the $D_{2}$ $\left(5 S_{1 / 2} \rightarrow 5 P_{3 / 2}\right)$ transition of rubidium, at a wavelength of $780 \mathrm{~nm}$. The input facet of the tapered gain element element has the typical width of a low power singletransverse mode diode laser $(\approx 5 \mu \mathrm{m})$. A seed beam is amplified in a single pass and expanded laterally by the taper to a width of typically $100-200 \mu \mathrm{m}$ [9] such that the light intensity at the output facet is kept below the damage threshold and the beam remains diffraction limited. The output power can be much larger than from a single-mode waveguide.

In previous work, TA's have been used as sources for frequency-doubling and pumping solid state lasers [11]. Apart from the achievable output power, frequency tunability of the narrow-linewidth output [12], simultaneous multifrequency generation [13], and spatial mode properties, including coupling to optical fibers [14] have been addressed.

In this paper we investigated the broadband spectral properties of the TA. The background due to amplified spontaneous emission (ASE) [15] in the gain element was minimized by adjusting the amplifier's operating conditions, i.e. temperature, injection current and seed input power. We also investigated the coupling efficiency of the TA output to a single-mode optical fiber, and find that the latter acts both as spatial and spectral filter. The properties of three gain elements of same type are compared.

Atom optical applications usually require good suppression of spectral background. E.g. in far off-resonance optical dipole traps [16], scattering of resonant light from the background causes heating and atom loss. We discuss the consequences of ASE background in such schemes. 


\section{AMPLIFIER SETUP}

Our amplifier system consists of a seed laser, the output beam of which is amplified in a single pass by the tapered gain element, as shown in Fig. 1. The TA output is coupled into a single-mode optical fiber [17]. The seed laser is an EGDL with a linewidth of less than $1 \mathrm{MHz}$. It contains a $60 \mathrm{~mW}$ single-mode laser diode (Hitachi, HL 7851 G98). From the EGDL we have $28 \mathrm{~mW}$ of power available to seed the amplifier at $780 \mathrm{~nm}$ wavelength. Coupling of the seeding beam to the amplifier is done by mode-matching the seed laser with the backwards travelling beam emitted by the TA. The divergence angles from seed laser emission and backward directed TA emission are similar. Hence, sufficient mode-matching is obtained using identical collimation lenses for both $(f=4.5 \mathrm{~mm}$, N.A. $=0.55)$. Additional mode shaping, e.g. with anamorphic prism pairs is not necessary. An optical isolator with $60 \mathrm{~dB}$ isolation protects the stabilized seed laser from feedback by the mode-matched beam of the amplifier. The $5 \mathrm{~mm}$ aperture of the isolator is sufficiently large that it does not clip the elliptical seed beam.

The TA was a SDL $8630 \mathrm{E}$ (ser.no. TD 310 18]). According to the manufacturer's data sheet the output power ranges from $0.5-0.55 \mathrm{~W}$ within a wavelength tuning range from $787-797 \mathrm{~nm}$, at an operating temperature of $21^{\circ} \mathrm{C}$. The beam quality parameter is specified as typically $M^{2}<1.4$ [18]. The TA should be protected from any reflected light, because it will be amplified in the backward direction and may destroy the entrance facet. We used an output collimator of large numerical aperture $(f=3.1 \mathrm{~mm}$, N.A. $=0.68)$ and sent the beam through a second $60 \mathrm{~dB}$ optical isolator. The plane of the tapered gain element is vertically oriented, so that diffraction yields a large horizontal divergence. This is collimated similar to the seed input, but yields a focus in the vertical plane. With a cylindrical lens $(f=100 \mathrm{~mm})$, we compensated for astigmatism of the beam, in order to couple into a single-mode optical fiber. The astigmatism correction is also shown in Fig. 1.

There is a considerable loss in optical power due to the isolator transmission. Taking also into account small reflection losses of the lens surfaces, we estimate the useful output power to be $78 \%$ of the optical power emitted by the TA facet. In the remainder of this paper, all quoted powers are as measured with a power meter behind the optical isolator.

The amplifier was provided as an open heatsink device. We mounted it on a water cooled base and stabilized it to the desired operating temperature within a few $\mathrm{mK}$ by a $40 \mathrm{~W}$ thermo-electric cooler. Thermal isolation from the ambient air and electromagnetic shielding were provided by a metal housing. When operating the amplifier at temperatures below the dew point, we flushed the containment with dry nitrogen.

It is necessary to have a compact, stable mounting of the gain element and collimators. We mounted the collimators in a commercial $x y$ flexure mount to allow for lateral lens adjustment. The axial $z$ adjustment is done by two small translation stages. All adjustments except the $z$ direction of the output collimator are accessible from outside. This proved to be very convenient for mode-matching the seed beam and also for compensating beam displacement of the TA output when changing temperature or current.

The narrow spectral line of seed laser and amplifier output was monitored by an optical spectrum analyzer of $1 \mathrm{GHz}$ free spectral range with $50 \mathrm{MHz}$ resolution. The amplifier's broad spectral background was analyzed using a grating spectrometer with a resolution of $0.27 \mathrm{~nm}$. Also the output of the single-mode fiber was recorded with the spectrometer.

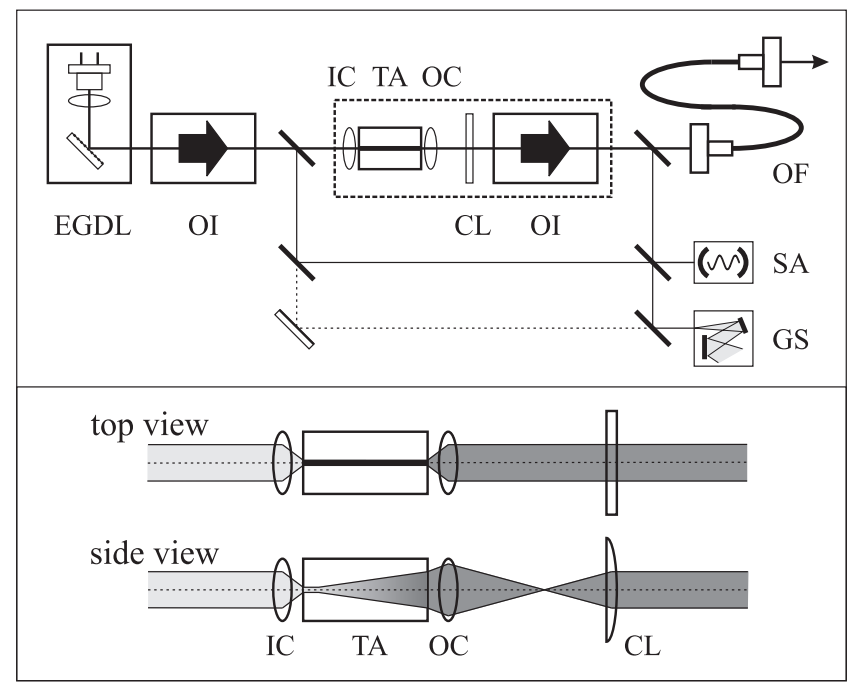

FIG. 1. Amplifier setup with seed laser and gain element: external grating diode laser (EGDL), $60 \mathrm{~dB}$ optical isolators (OI), tapered amplifier (TA), single-mode optical fiber (OF), optical spectrum analyzer (SA), and grating spectrometer (GS). A top and side view of the gain element is shown with input and output collimators (IC,OC), and a cylindrical lens (CL) compensating for astigmatism (not to scale).

\section{UNSEEDED OPERATION OF THE AMPLIFIER}

When the TA receives no seed input, it operates as a laser diode. Thus, when the injection current, $I_{T A}$, is increased from zero, the optical output power shows the lasing threshold (see Fig. 3ab). Generally, both the operating wavelength and the optical power of a laser diode depend on the temperature. This property is shown in Fig. 2. The emission spectrum of the lasing tapered gain element is almost Gaussian shaped, with an $1 / e^{2}$ width of $4 \mathrm{~nm}$. It appears as a background of ASE also in the spectra when operating the gain element as an amplifier (see below). The oscillatory structures on the spectra are artifacts of the spectrometer. In the fitted spectra, 
we evaluated the center wavelength at each temperature setting. It increases with temperature with a slope of $0.28 \mathrm{~nm} / \mathrm{K}$, typical for semiconductor lasers (Fig. 2b).

The temperature dependence of the output power is shown in Fig. 2 2 c. We operated the TA within the specifications of the manufacturer's data sheet, that recommends to keep the optical power at the output facet below $550 \mathrm{~mW}$. As the temperature increases, the conversion efficiency $(\mathrm{mW} / \mathrm{A})$ decreases and the threshold current increases. This can be seen in Fig. Bab (open symbols) where we plot the optical output power $P$ vs. current $I_{T A}$ for two temperature settings. The threshold current of the unseeded TA increases from $0.78 \mathrm{~A}\left(5^{\circ} \mathrm{C}\right)$ to $0.86 \mathrm{~A}\left(14^{\circ} \mathrm{C}\right)$. From the slopes above threshold, we find that the conversion efficiency decreases from $0.7 \mathrm{~W} / \mathrm{A}$ $\left(5^{\circ} \mathrm{C}\right)$ to $0.5 \mathrm{~W} / \mathrm{A}\left(14^{\circ} \mathrm{C}\right)$. In order to measure the unperturbed output of the unseeded TA, one has to prevent light emitted from the entrance facet of being reflected. Even a very weak reflection would be amplified in the forward direction.

For the unseeded TA, we also measured the light propagating backwards from the amplifier's entrance facet. It reaches typically a power of $10-25 \mathrm{~mW}$ for injection currents from $1-1.4$ A. Hence the necessity of a good isolation of the seeding laser.
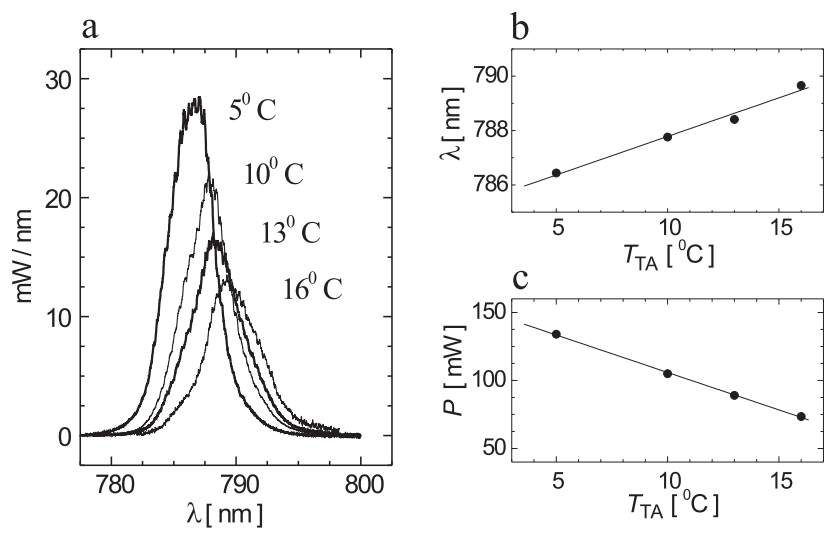

FIG. 2. Temperature dependence of the unseeded amplifier at 1.2 A injection current: (a) spectra, (b) center wavelengths, (c) output power after optical isolator. Solid lines indicate linear fits.

\section{AMPLIFICATION OF A SEED BEAM}

Amplification of a seed beam is evident in the output power of the TA. In Fig. 3ab, we have plotted the output power for distinct seed powers, $P_{\text {seed }}$, at two temperature settings. For the larger seed inputs of $8.6 \mathrm{~mW}$ and $5.3 \mathrm{~mW}$, respectively, the amplifier was well saturated. The saturation is evident from Fig. 3c where $P_{\text {seed }}$ was varied for injection currents from $0.8-1.3 \mathrm{~A}$.
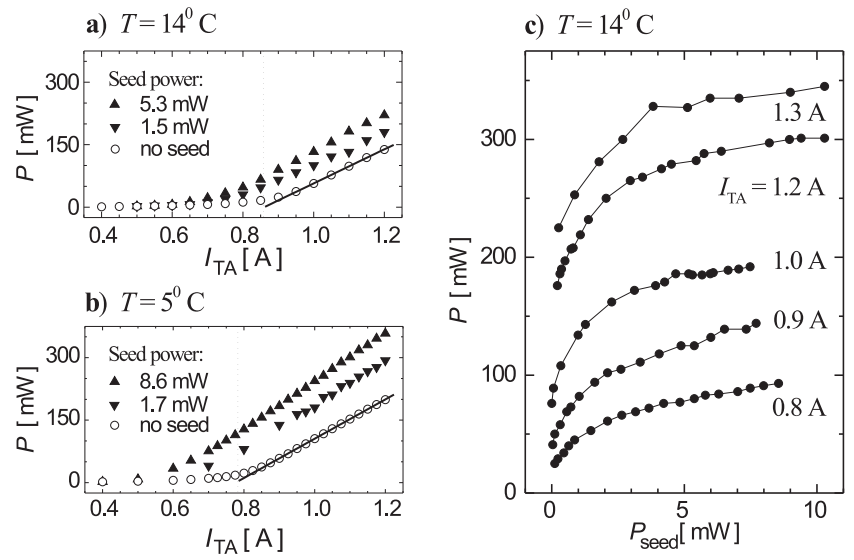

FIG. 3. Amplifier output vs. injection current $I_{T A}$ and seed power $P_{\text {seed }}$. The lasing thresholds for the unseeded amplifier are $0.86 \mathrm{~A}\left(14^{\circ} \mathrm{C}\right)$ and $0.78 \mathrm{~A}\left(5^{\circ} \mathrm{C}\right)$, indicated by vertical dotted lines.

With $P_{\text {seed }} \approx 4 \mathrm{~mW}$ the device appears to be saturated for all current settings. For seed powers between $2-4 \mathrm{~mW}$, the amplification ranges from $70-140$, e.g. $320 \mathrm{~mW}$ output with $4 \mathrm{~mW}$ seed.

We now discuss the spectral properties of the TA and in particular the suppression of ASE background. Fig. 4 shows the power spectral density of the TA output before an optical fiber for $16^{\circ} \mathrm{C}$ and $5^{\circ} \mathrm{C}$ operating temperature. In both cases the amplifier was saturated with $28 \mathrm{~mW}$ seed input. For comparison also the corresponding spectra of the unseeded amplifier are shown. In saturation, the broad ASE background is distinguished from a narrow peak of the amplified seed signal. The width of the peak is given by the bandwidth of the spectrometer, $0.27 \mathrm{~nm}$ FWHM. (By means of an optical spectrum analyzer and Doppler-free spectroscopy signals of rubidium, we could verify that the amplified signal has a narrow width comparable to that of the EGDL.)

The influence of the operating temperature is obvious first by the increased output power at lower temperature: $323 \mathrm{~mW}\left(16^{\circ} \mathrm{C}\right)$ and $410 \mathrm{~mW}\left(5^{\circ} \mathrm{C}\right)$, respectively. Second, both the peak level and total amount of ASE background are better suppressed at lower temperature. We attribute this to the shift of the gain profile of the TA towards the seed wavelength of $780 \mathrm{~nm}$ at lower temperature [15]. The fraction of ASE background in the TA output is obtained by integrating the power spectral densities in Fig. 团, yielding $5.6 \%\left(16^{\circ} \mathrm{C}\right)$ and $1.4 \%\left(5^{\circ} \mathrm{C}\right)$, respectively.

More than the total ASE fraction, for atom-optical applications the important figure is the fraction of ASE within the natural linewidth of the atomic transition used. We define this ratio $\epsilon$ by comparing the power in the peak with the ASE power in a bandwidth given by a typical atomic natural linewidth, e.g. $\Gamma / 2 \pi=$ $6 \mathrm{MHz}$ for rubidium. For $16^{\circ} \mathrm{C}\left(5^{\circ} \mathrm{C}\right)$, the peak value 
of $+2.5 \mathrm{dBm} / \mathrm{nm}(-2.0 \mathrm{dBm} / \mathrm{nm})$ of $\mathrm{ASE}$ is then reexpressed as $22 \mathrm{nW} / \Gamma(7.9 \mathrm{nW} / \Gamma)$. With $323 \mathrm{~mW}$ $(410 \mathrm{~mW})$ in the narrow line, the suppression ratio $\epsilon$ is $-72 \mathrm{~dB}(-77 \mathrm{~dB})$. We can thus optimize the spectral properties of the TA output by an appropriate choice of operating temperature. Even better suppression can be achieved by use of an optical fiber as spectral filter, as discussed in the following section.
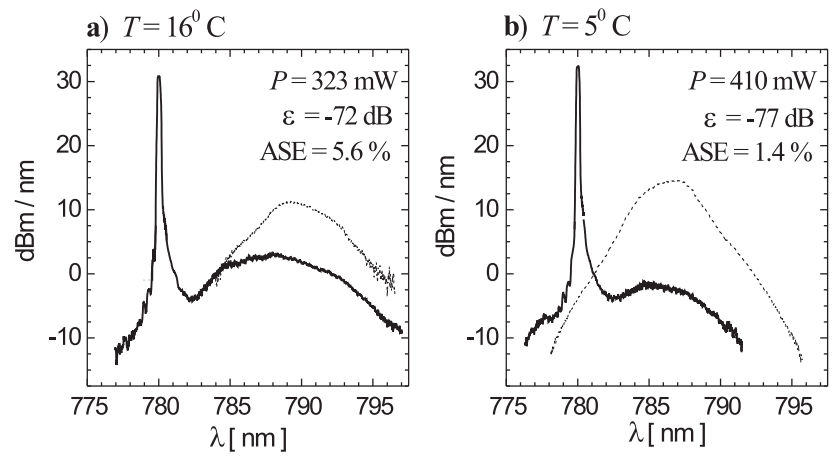

FIG. 4. Spectrum of the amplifier output (before the fiber). The seed power is $28 \mathrm{~mW}$ at $1.2 \mathrm{~A}$ injection current. The dashed curves are for unseeded operation. $P$ is the total optical power, ASE is the fraction of background power, and $\epsilon$ is the ASE suppression for the power spectral density in units of $\mathrm{mW} / \Gamma(\Gamma / 2 \pi=6 \mathrm{MHz})$.
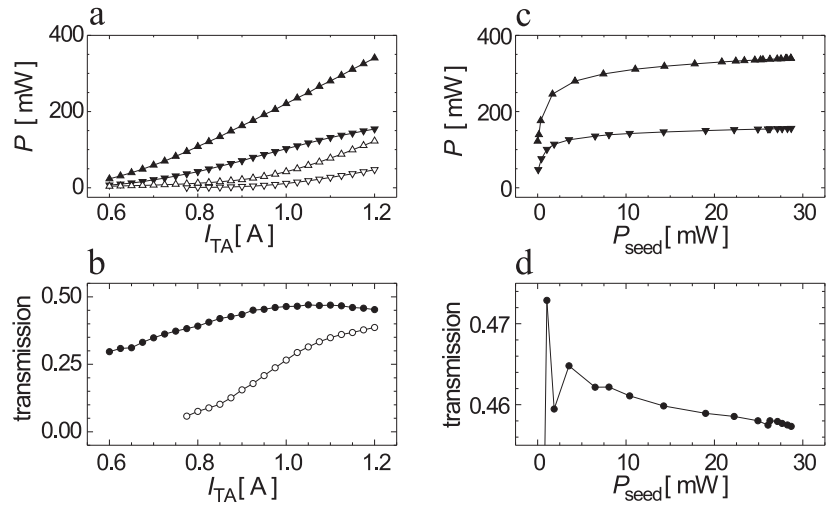

FIG. 5. Transmission through a single-mode optical fiber. (a) Fiber input and output power with and without seed, (b) fiber transmission with and without seed, (c) Fiber input and output in dependence on seed power, (d) corresponding fiber transmission. The symbols: seeded with $28 \mathrm{~mW}$ (solid), unseeded (open), fiber input (up triangles), fiber output (down triangles), fiber transmission (circles).

\section{SPATIAL AND SPECTRAL FILTERING USING AN OPTICAL FIBER}

For many applications, laser beam quality is an important property, e.g. for optical dipole traps. A convenient method to obtain spatial filtering is to send the light through a single-mode optical fiber. An additional advantage of the fiber is a decoupling of optical alignment between different parts of the experimental setup. Here the coupling efficiency is discussed and the spectrum of the transmitted light is compared with the spectrum before the fiber. We observe that spatial filtering by the fiber is accompanied by spectral filtering. Evidently, the contribution of ASE in the TA beam is spatially distinguishable from the amplified seed signal.

We find that the spatial mode properties of the saturated TA output are slightly different for different injection currents. Fig. 5 ab represents the fiber transmission vs. current, $I_{T A}$. The fiber coupling was optimized for a current of $1 \mathrm{~A}$ and the TA was saturated. A maximum transmission of $46 \%$ is achieved. For comparison, with an EGDL, after circularizing the beam using an anamorphic prism pair, we typically obtain a fiber transmission of $75 \%$. The slope in the transmission curve is probably due to a beam displacement caused by the current-dependent thermal load of the gain element. Such a displacement was also observed when the operating temperature was changed. With the fiber coupling thus optimized, light from the unseeded TA has less transmission than the amplified seed signal. Fig. 5cd shows for a fixed current of $1 \mathrm{~A}$, that the fiber transmission is almost independent of the seed input power, i.e. the beam shape does not change.

Also the light after the fiber has been analyzed using the grating spectrometer, see Fig. 6a for an operating temperature of $5^{\circ} \mathrm{C}$. For the saturated amplifier a spectral ASE background cannot be distinguished after the fiber, since the peak is identical with the spectrometer response function. (This response function was obtained by recording the spectrum of the narrow-linewidth EGDL laser. A similar response was also obtained using a HeNe laser.) Thus we can only give an upper limit for the ASE contribution of $0.2 \%$. The suppression ratio is $\epsilon<-82 \mathrm{~dB}$, with an ASE level of less than $-12.5 \mathrm{dBm} / \mathrm{nm}$ or $0.7 \mathrm{nW} / \Gamma$, respectively. This should be compared to the value of $\epsilon=-77 \mathrm{~dB}$ before the fiber, as seen in Fig. $4 \mathrm{~b}$ for $5^{\circ} \mathrm{C}$. For comparison, at $16^{\circ} \mathrm{C}$, we found an ASE suppression of only $-76 \mathrm{dBm}$ after the fiber.

The ASE background depends also on the degree of amplifier saturation, as shown in Fig. 6 b. The ASE fraction is plotted vs. seed power for light before and after the fiber. It decreases quickly as the TA saturates. From the spectra acquired before the fiber (up triangles), it is evident that the increase of seed power into the saturated regime suppresses the ASE. Whereas mode matching of the seed input beam was not difficult for achieving max- 
imum output power, optimal ASE suppression required a more careful alignment, thus optimizing the TA saturation.

It is also obvious from the figure, that larger gain of the TA improves the output spectrum (circles, larger operating current).

We can summarize the results of Sec. IV and V as follows: The spectral properties of the TA can be optimized by choosing an appropriate operating temperature, spectral filtering with an optical fiber and saturation of the gain element.
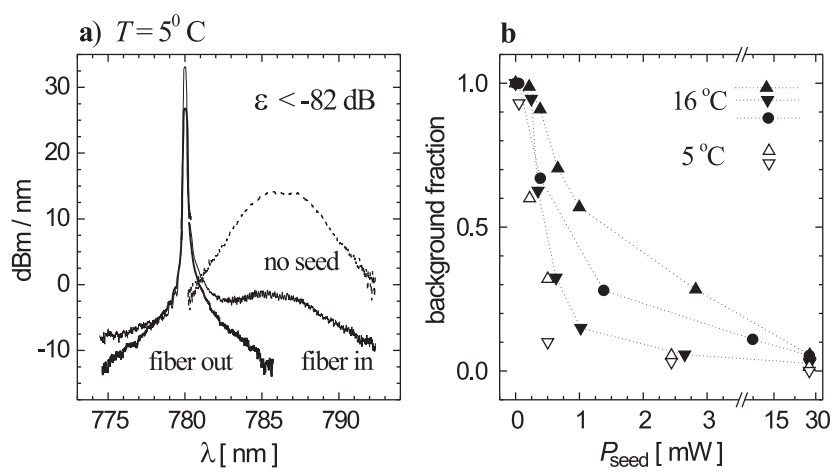

FIG. 6. Spectral filtering by a single-mode optical fiber. (a) Saturated with $28 \mathrm{~mW}$ seed power at 1.2 A current, $130 \mathrm{~mW}$ power after the fiber, $410 \mathrm{~mW}$ in front of it. ASE background is not distinguishable from the spectrometer response function after the fiber. (b) The ASE fraction depends on the saturation: fiber input (up triangles) and output (down triangles) at $1.2 \mathrm{~A}$ current. For comparison: fiber input with 1.45 A current (circles).

\section{VARIATION IN THE PROPERTIES OF INDIVIDUAL GAIN ELEMENTS}

We compared the gain element with two other gain elements of the same type $(8630 \mathrm{E})$. One gain element (ser. no. TD 430, $777 \mathrm{~nm}$ ) was used in the setup described above. A second (ser. no. TD 387, $790 \mathrm{~nm}$ ) was implemented in a commercial TA system (TUI Optics $G m b H$, TA 100) and operated on both the $D_{2}$ and the $D_{1}\left(5 S_{1 / 2} \rightarrow 5 P_{1 / 2}\right)$ transition of rubidium at $780 \mathrm{~nm}$ and $795 \mathrm{~nm}$, respectively.

For the different gain elements, we found considerable differences in their beam quality and consequently their fiber coupling efficiency. Whereas TD 310 and TD 387 showed a dominant double-lobed mode structure in the far field and permitted only a fiber transmission of $46 \%$, the TD 430 beam showed a less pronounced lobe structure 20]. With this gain element, we could couple $59 \%$ to the fiber and obtained $200 \mathrm{~mW}$ of optical power after the fiber and an ASE suppression of better than $-84 \mathrm{dBm}$.

Also the amplification properties showed striking dif- ferences among the gain elements. TD 430 has similar saturation properties as TD 310. In contrast, TD 387 operates at maximum output power already without seed. This may be due to different antireflection coatings at the TA facets. Hence, the TD 387 requires (permanent) monitoring by a spectrometer in order to optimize seed incoupling and ASE suppression. The current of the TD 387 cannot be tuned continuously, because it shows discrete "locking-ranges", resembling the injection-locking behavior of single-mode diode lasers.

\section{FAR OFF-RESONANCE DIPOLE POTENTIALS WITH SPECTRAL BACKGROUND}

In this section we present an estimate of the consequences of the broad spectral ASE background for light scattering in optical dipole traps. A background that covers atomic resonances, leads to extra resonant scattering. Usually the detuning $\delta$ for a dipole trap is chosen as large as possible, given the available laser intensity $I$. The reason is that off-resonance scattering scales as $\Gamma_{O R}^{\prime} \propto I / \delta^{2}$ at low saturation and large detuning, whereas the dipole potential is only inversely proportional to the detuning, $\mathcal{U} \propto I / \delta[21$.

In the presence of a resonant background the total scattering rate of the atoms is $\Gamma^{\prime}=\Gamma_{O R}^{\prime}+\Gamma_{R}^{\prime}$, where $\Gamma_{R}^{\prime}$ represents the resonant scattering. For a fixed depth of the optical dipole potential this results in a maximum useful laser detuning, $\delta_{\max }$, at which the scattering rate of the atoms, $\Gamma^{\prime}$, is minimized.

With low atomic saturation by a weak spectral background, we can write $\Gamma_{R}^{\prime} \approx(\Gamma \pi / 4) \epsilon I / I_{0}$. Here $I_{0}$ is the saturation intensity, i.e. $1.6 \mathrm{~mW} / \mathrm{cm}^{2}$ for the $D_{2}$ line of rubidium. Hence, with the requirement of a fixed potential $\mathcal{U}$, the two scattering contributions scale as $\Gamma_{O R}^{\prime} \propto 1 / \delta$ and $\Gamma_{R}^{\prime} \propto \delta$, respectively. This results in the optimum detuning and minimum scattering rate,

$$
\begin{aligned}
\delta_{\max } & = \pm \Gamma / \sqrt{2 \pi \epsilon}, \\
\Gamma^{\prime} & =2 \sqrt{2 \pi \epsilon} \mathcal{U} / \hbar .
\end{aligned}
$$

As an example we consider atoms cooled to a temperature of a few $\mu \mathrm{K}$ in optical molasses and require an optical potential depth of $\mathcal{U} / h \approx 1 \mathrm{MHz}$. If the allowable scattering rate is, e.g. $\Gamma_{\max }<100 \mathrm{~s}^{-1}$, this yields a required background suppression $\epsilon<-110 \mathrm{~dB}$ and optimum detuning $\delta_{\max } \approx 760 \mathrm{GHz}$. Such a small background contribution is of course beyond the resolution of our spectrometric data, with which we observe at best an upper limit of $I_{R}(\delta)<0.7 \mathrm{nW} / \Gamma$ for a total optical power of $200 \mathrm{~mW}$. This corresponds to a background suppression of $\epsilon<-84 \mathrm{~dB}$. With a detuning of $760 \mathrm{GHz}$, the extension of the optical potential is restricted to less than $250 \mu \mathrm{m}$. 


\section{CONCLUSIONS}

We have investigated a tapered semiconductor amplifier system, that provides 150-200 $\mathrm{mW}$ narrow linewidth output from a single-mode optical fiber, where the fiber transmission is up to $59 \%$, depending on the actual gain element in use. The system requires less than $5 \mathrm{~mW}$ seed input to saturate with an amplification up to 140 at this seed level. The output of the amplifier includes a broad spectral background of amplified spontaneous emission. We have found three means of reducing this background: (i) Choosing the operating temperature such that the gain profile of the amplifier is spectrally centered as close as possible to the amplified wavelength, (ii) spectrally filtering the output beam with a single-mode optical fiber, and (iii) saturating the amplifier with sufficient seed input power. With those measures, the ASE background is below the resolution of our spectrometer. That is, the ASE fraction is less than $0.2 \%$ of the optical power in the beam and the peak level is less than $0.1 \mathrm{nW} / \mathrm{MHz}$. Relating the power spectral density of the background to the natural transition linewidth of rubidium $(\Gamma / 2 \pi=6 \mathrm{MHz})$, the ASE suppression is better than $-82 \mathrm{~dB}$.

We discussed the atom optical application of such an amplifier system with far off-resonance dipole potentials. A broad ASE background implies here an optimum laser detuning with which light scattering by atoms is minimized.

A tapered amplifier system may be a lower-cost option to a Ti:Sapphire laser. The available single-transverse mode optical power and spectral properties are similar to those of broad-area semiconductor laser amplifiers.

\section{ACKNOWLEDGEMENTS}

We wish to thank K. Dieckmann, A. Görlitz, W. Kaenders, J. Schuster, I. Shvarchuck, B. Wolfring, A. Zach, and C. Zimmermann for helpful information. This work is part of the research program of the "Stichting voor Fundamenteel Onderzoek van de Materie" (Foundation for the Fundamental Research on Matter) and was made possible by financial support from the "Nederlandse Organisatie voor Wetenschappelijk Onderzoek" (Netherlands Organization for the Advancement of Research). R.S. has been financially supported by the Royal Netherlands Academy of Arts and Sciences.

[1] S. Chu: Rev. Mod. Phys. 70, 685 (1998)

C.N. Cohen-Tannoudji: ibid. 70, 707 (1998)

W.D. Phillips: ibid. 70, 721 (1998)

C.S. Adams, E. Riis: Prog. Quant. Electr. 21, 1 (1997)
[2] C.E. Wieman, L. Hollberg: Rev. Sci. Instrum. 62, 1 (1991)

[3] G.R. Hadley: IEEE J. Quant. Electr. QE-22, 419 (1986)

P. Spano, Salvatore Piazzolla, Mario Tamburrini: IEEE J. Quant. Electr. QE-22, 427 (1986)

[4] G.L. Abbas, S. Yang, V.W.S. Chan, J.G. Fujimoto: IEEE J. Quant. Electr. QE-24, 609 (1988)

L. Goldberg, M.K. Chun: Appl. Phys. Lett. 53, 1900 (1988)

[5] L. Goldberg, D. Mehuys, M.R. Surette, D.C. Hall: IEEE J. Quant. Electron. QE-29, 2028 (1993)

[6] A.C. Fey-den Boer, H.C.W. Beijerinck, K.A.H. van Leeuwen: Appl. Phys. B 64, 415 (1997)

E. Gehrig, B. Beier, K.-J. Boller, R. Wallenstein: Appl. Phys. B 66, 287 (1998)

M. Praeger, V. Vuletic, T. Fischer, T.W. Hänsch, C. Zimmermann: Appl. Phys. B 67, 163 (1998)

[7] I. Shvarchuck et al.: private communication

[8] K. Iida, H. Horiuchi, O. Matoba, T. Omatsu, T. Shimura, K. Kuroda: Opt. Commun. 146, 6 (1998)

[9] J.N. Walpole: Opt. Quant. Electr. 28, 623 (1996)

[10] J.N. Walpole, E.S. Kintzer, S.R. Chinn, C.A. Wang, L.J. Missaggia: Appl. Phys. Lett. 61, 740 (1992)

D. Mehuys, D.F. Welch, L. Goldberg: Electron. Lett. 28, 1944 (1992)

[11] C. Zimmermann, J. Walz, T.W. Hänsch: Hyperfine Interactions 100, 145 (1996)

[12] D. Wandt, M. Laschek, F. v. Alvensleben, A. Tünnermann, H. Welling: Opt. Commun. 148, 261 (1998)

[13] G. Ferrari, M.-O. Mewes, F. Schreck, C. Salomon: Opt. Lett. 24, 151 (1999)

[14] J.C. Livas, S.R. Chinn, E.S. Kintzer, J.N. Walpole, C.A. Wang, L.J. Missaggia: IEEE Phot. Techn. Lett. 6, 422 (1994)

Z.L. Liau, J.N. Walpole, D.E. Mull, C.L. Dennis, L.J. Missaggia: Appl. Phys. Lett. 64, 3368 (1994)

Z.L. Liau, J.N. Walpole, J.C. Livas, E.S. Kintzer, D.E. Mull, L.J. Missaggia, W.F. DiNatale: IEEE Phot. Techn. Lett. 7, 1315 (1995)

[15] W.W. Chow, R.R. Craig: IEEE. J. Quant. Electron. QE26, 1363 (1990)

[16] R. Grimm, M. Weidemüller, Yu.B. Ovchinnikov: Adv. At. Mol. Opt. Phys. 42, 95 (2000)

[17] OFR Inc., fiber port PAF-X-5-780, input beam diameter $0.9-1.8 \mathrm{~mm}$

[18] Spectra Diode Laboratories, San Jose, Calif.: Product Catalog $(1996 / 97)$

[19] TUI Optics GmbH: Diode tapered amplifier system TA 100 (catalog 1997, data sheets 1999)

[20] From the first operation on, the TD 310 output showed a shadow in the near field. After approximately 100 hours of operation the gain element quickly degraded and became inoperable.

[21] C.N. Cohen-Tannoudji, J. Dupont-Roc, and G. Grynberg: Atom-Photon Interactions (Wiley, New York, 1992) 